\title{
COMPARISON OF CASTING AND SOLIDIFICATION OF 12 TON STEEL INGOT USING TWO DIFFERENT NUMERICAL SOFTWARE
}

\author{
Ondřej KOTÁSEK, Vladislav KURKA, Marek VINDYŠ, Petr JONŠTA, Roman NOGA, \\ Marek DOBIÁŠ \\ MATERIAL AND METALLURGICAL RESEARCH, Ltd., Ostrava, Czech Republic, projekty@mmvyzkum.cz
}

https://doi.org/10.37904/metal.2021.4091

\begin{abstract}
The submitted article deals with the study of casting and solidification of a $12 \mathrm{t}$ ingot made of structural steel $42 \mathrm{CrMo} 4$ using numerical simulations in the THERCAST® and MAGMASOFT $®$ software. The possibilities of each individual software in filling and solidification of steel were assessed, including the prediction of ingot defects such as porosity, shrinkage cavities or shrinkage porosities; the proportion of liquid phase, segregation of selected elements and solidification time were also monitored.. The results of the numerical simulations indicated that THERCAST ${ }^{\circledR}$ allows easier use of higher types of approximation of the function sought in order to increase the accuracy of the solution. If a part of the model needs to be defined more precisely, THERCAST® software can achieve this with local refinement, while places with simple geometry can be described using a coarser mesh. Unlike MAGMASOFT ${ }^{\circledR}$ in the basic version, THERCAST $®$ has the possibility to simulate various behaviours of the material based on one geometric model, including, e.g., deformation, stress, density.
\end{abstract}

Keywords: Numerical simulation, THERCAST®, MAGMASOFT®, steel ingot, casting, solidification

\section{INTRODUCTION}

Numerical simulations are a very important and useful tool in verifying and optimizing modern metallurgical processes. They allow us to predict, for example, the characteristics of steel flow in production units, temperature profiles, steel crystallization, the occurrence of defects, stress states in the ingot, the distribution of elements along the height and cross-section of the casting, etc. [1,2]. Some processes studied based on numerical simulations would also be almost unsolvable under the operating conditions of a steel plant.

Numerical methods are based on the discretization of variables, which is supported by the typical repeatability of simple algebraic operations of a certain type. Numerical methods allow us to obtain the solution to a given thermal problem in a finite number of discrete points, both in the whole area and in the surface part only. The tasks are solved using one of two numerical methods - either the Finite element method (FEM) or the Finite difference method (FDM) [3].

FEM is a numerical method for solving (partial) differential equations in the continuum. It uses spatial discretization mostly by so-called triangulation of the area solved, where a continuous function is sought in parts. The boundaries of the area solved is then replaced by polynomials. The basic elements describing the surface are triangular or quadrangular spots. In spatial representation, these are prisms, tetrahedrons or pyramids [3] a [4].

FDM, also referred to as the mesh method, is based on the approximation of the basic differential equation with the specific boundary conditions corresponding to the differential equation, which has the form of algebraic equations. The perfection of the approximation consists of replacing the derivative with more precise 
expressions. The replacement is performed at discrete locations formed by mesh nodes that include the area being studied. The final result of algebraic operations is to determine the temperature at a given mesh node.[3]

Due to the rapid development of computer technology at the beginning of the third millennium, various commercial simulation software tools are now widely available. These software tools are usually designed on the basis of various modular solutions focusing on a specific technological process and related quantities. Numerical calculation in commercial software generally consists of the following phases: preprocessing includes the creation of geometry and generation of the computing mesh, processing - definition and solution of the calculation itself, post-processing - analysis of the results of the numerical simulation $[5,6]$.

At the company MATERIÁLOVÝ A METALURGICKÝ VÝZKUM s.r.o. (hereinafter referred to as MMV), the MAGMASOFT $®$ v5.4.1.0 software from the German company MAGMA and THERCAST® NxT 2.0 software from the French company TRANSVALOR are currently used for numerical simulations of casting and solidification of melt steel.

THERCAST $®$ is a computer software tool that works on the basis of the finite element method (FEM). It is intended for foundry processes, continuous casting and ingot casting. THERCAST® allows us to accurately analyse various production processes from the beginning of casting to the end of the solidification phase. It may be used to study the thermal field of the metal object and some surrounding components during the process. It is able to determine flow rates, pressure, stress or deformation in metal. Based on this, it can calculate the final shape and properties of the casting. A characteristic feature of THERCAST $\circledast$ is its ability to predict the real behaviour of the material in the mould and during the gas evolution during the process. It predicts results verified by real processes thanks to an accurate thermo-mechanical model. The software also contains a powerful solver of systems of vector equations for the efficient use of computing power using parallel calculations. Calculations in this software are divided into thermal, thermo-hydraulic and thermo-hydraulic + thermo-mechanical parts [7].

MAGMASOFT $®(M A G M A)$ is a comprehensive and effective simulation tool for improving casting quality, optimizing process conditions and significantly reducing foundry costs. It is a simulation program based on the simulation of heat flow, flow dynamics, structural changes during solidification when considering real casting conditions. MAGMA can simulate both large solid steel castings made by the gravity casting method and small castings made of magnesium, copper and aluminium alloys, MMV includes the MAGMAsteel module. To solve various types of casting methods, MAGMA uses a number of additional modules that allow all parameters of a specific casting process to be set 0 . The main modules are as follows:

- MAGMAsteel - simulation of convection during solidification, prediction of segregation of main alloying elements

- $\quad$ MAGMAhpdc - high-pressure casting module

- MAGMAlpdc - low-pressure casting module

- MAGMAstress - stress solution module

In order to assess the possibilities of both software solutions, the casting and solidification of a $12 \mathrm{t}$ round ingot made of $42 \mathrm{CrMo} 4$ structural steel was numerically simulated. Attention was focused on the filling and solidification of the steel, including the prediction of ingot defects such as porosity, shrinkage cavities or shrinkage porosities. Other studied variables were the proportion of liquid phase, segregation of carbon, chromium, molybdenum and manganese, metal flow and solidification time.

\section{NUMERICAL SIMULATION SETTINGS}

The THERCAST NxT 2.0 and MAGMA v5.4.1.0 software performed a numerical simulation of the bottom casting of a 12 t round ingot into an ingot mould. Figure 1 shows the ingot geometry and the selected mesh. 
The ingot was meshed in the THERCAST software to an element size of $0.008 \mathrm{~m}$ and in the MAGMA software the volume mesh consisted of 521,024 cubes. The casting speed was the same for both simulations $(0.001217$ $\mathrm{m}^{3} / \mathrm{s}$ ) and the casting temperature was $1536^{\circ} \mathrm{C}$. The accuracy of the results of the numerical simulations of defects inside the ingot, especially macrosegregation, is determined by the quality of the thermodynamic conditions, steel models, and definition of the heat transfer coefficient between individual materials.
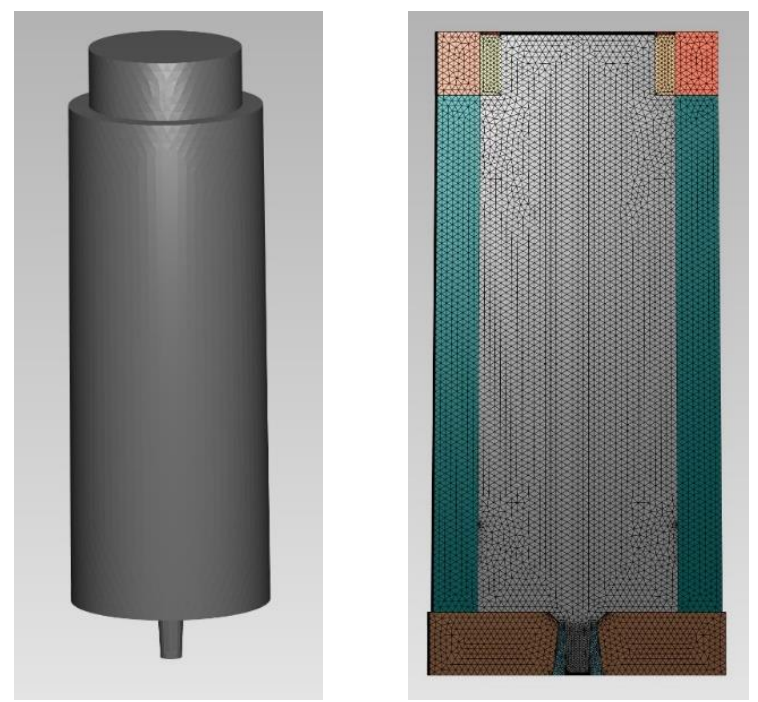

The properties of the refractory materials and heat transfer coefficients, including material models, were therefore intentionally used from the THERCAST and MAGMA libraries, respectively. 42CrMo4 steel was chosen for the numerical simulations. It is a low-alloy stainless chrome-molybdenum steel intended for tempering. The chemical composition of the 42CrMo4 steel used for the numerical simulations is given in Table 1.

Figure $13 \mathrm{D}$ ingot geometry and the selected mesh

Table 1 Chemical composition of $42 \mathrm{CrMo} 4$ steel used for numerical simulations (wt. \%)

\begin{tabular}{|c|c|c|c|c|c|c|c|}
\hline Element & $\mathbf{C}$ & $\mathbf{M n}$ & $\mathbf{S i}$ & $\mathbf{P}$ & $\mathbf{S}$ & $\mathbf{C r}$ & Mo \\
\hline $\mathbf{W t} \%$ & 0.4 & 0.7 & 0.1 & 0.015 & 0.010 & 1.1 & 0.2 \\
\hline
\end{tabular}

\section{DISCUSSION OF RESULTS}

The temperature field of the metal during the filling of the mould is documented in Figure 2, the segregation of selected elements from the filling of the ingot to the time of cooling is shown in Figures 3, 4.

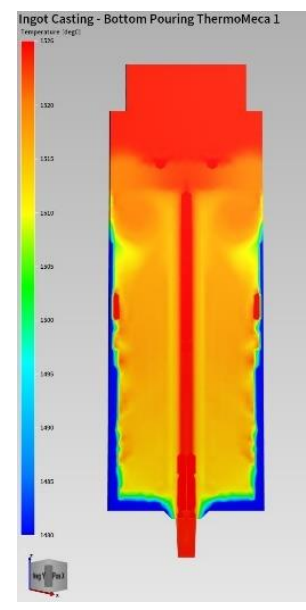

$75 \%$ filling

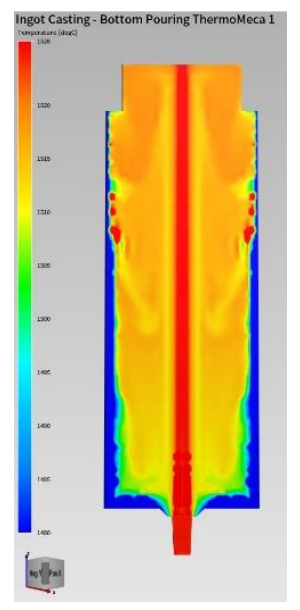

$100 \%$ filling

a) THERCAST
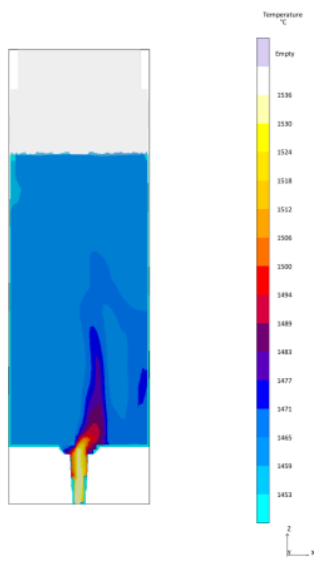

man

$75 \%$ filling
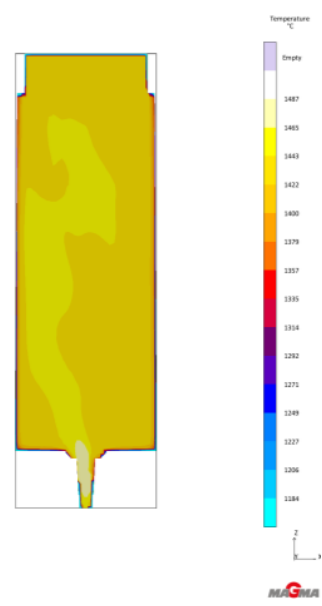

$100 \%$ filling

b) MAGMA

Figure 2 Temperature field of metal during mould filling 
From Figure 2 it can be seen at first glance that THERCAST (FEM) simulates, despite the turbulent flow, completely symmetrically, while MAGMA (FDM) gives non-symmetrical results. This may be related to the fact that MAGMA uses cube-shaped elements, which, in case the body is not rectangular, leads to a different approximation of the shapes of the simulated body. For liquid metals, MAGMA expects slightly lower temperatures than THERCAST, which influences the total solidification time, which was $2.75 \mathrm{~h}$ based on the THERCAST calculation and $2.57 \mathrm{~h}$ based on the MAGMA calculation. In solid ingots, both microsegregation and macrosegregation generally occur due to the longer solidification time. Figure 3 shows the segregations of carbon, chromium, manganese and molybdenum based on the MAGMA software, and Figure 4 shows identical segregations based on the THERCAST software, which defines the segregation according to Equation 1. Compared to THERCAST, MAGMA again shows an asymmetrical distribution of segregations.

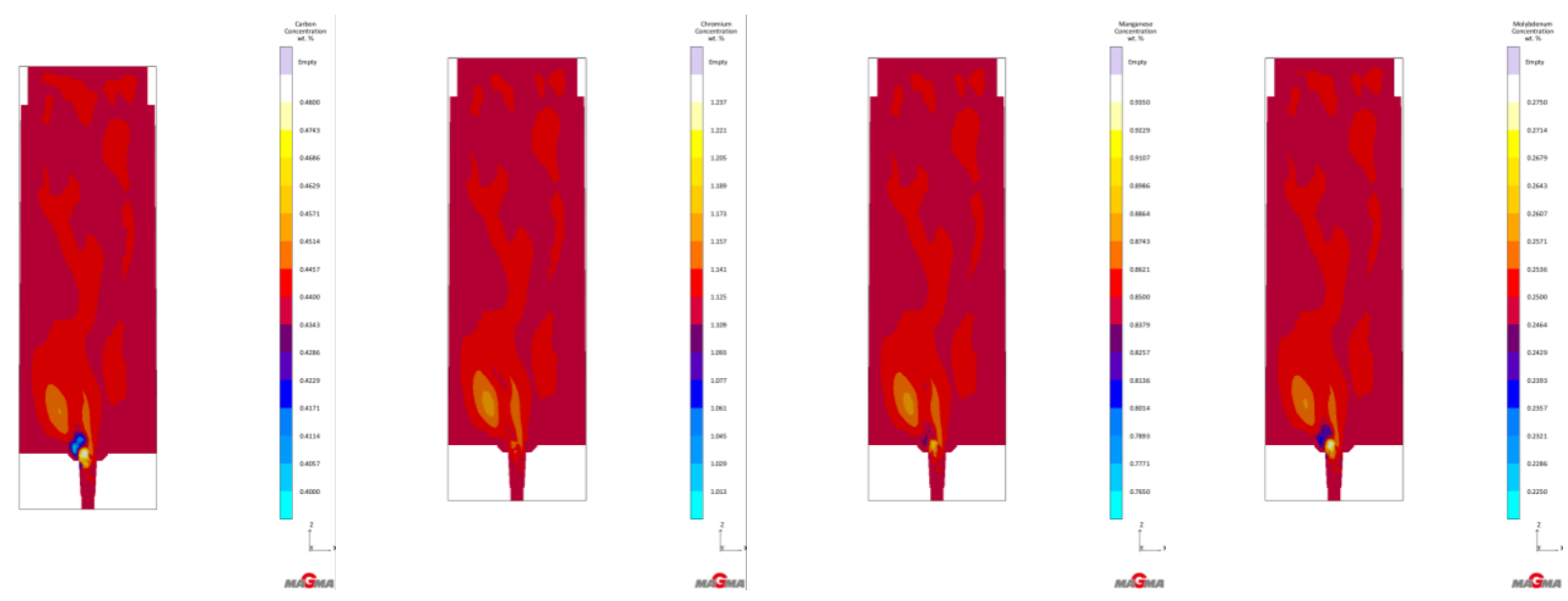

Figure 3 Segregation of carbon, chromium, manganese and molybdenum in MAGMA software (wt \%)

$\operatorname{seg}=\frac{\left(w_{x}-w_{x}^{0}\right)}{w_{x}^{0}}$

where:

$w_{x}$ - local concentration of a specific element (-)

$w_{x}^{0}-$ initial concentration of a specific element (-)
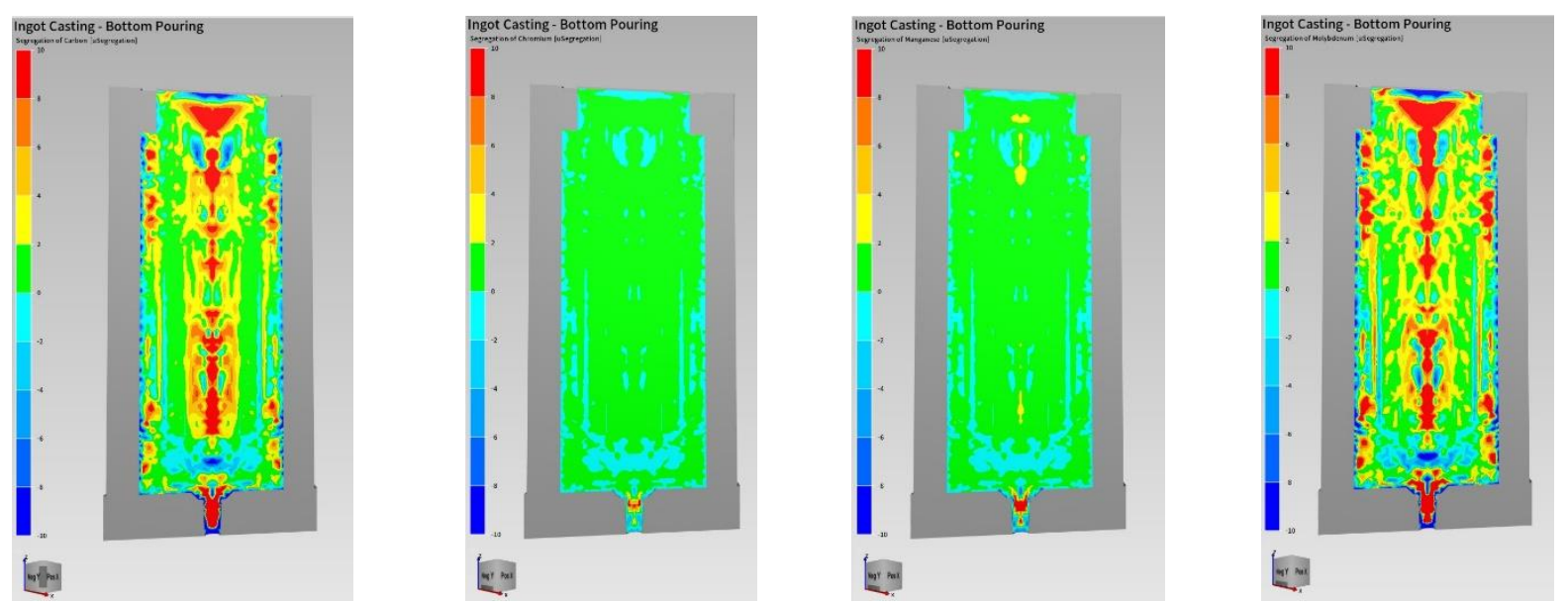

Figure 4 Segregation of carbon, chromium, manganese and molybdenum in THERCAST software 
The prediction of microporosity formation according to the Niyama criterion is documented in Figure $\mathbf{5}$ and Figure 6. Both the MAGMA and THERCAST software tools calculated the greatest risk of microporosity in the central area, with an increased possibility of occurrence in the ingot head. The number of critical points was lower around the perimeter and in the inlet part of the ingot. The difference in results by 3 orders is due to the fact that THERCAST displays the results in the units of $\frac{m}{\left({ }^{\circ} C \cdot s\right)^{1 / 2}}$ and MAGMA in the units of $\frac{m m}{\left({ }^{\circ} C \cdot s\right)^{1 / 2}}$. It can be stated that the results of microporosity are very similar for both simulations.

The Suzuki criterion represents what is called ghost lines, i.e. a segregation. According to this criterion, it was possible to perform the simulation only in THERCAST, see Figure 6 . The occurrence of A-segregation was predicted only in the head part.

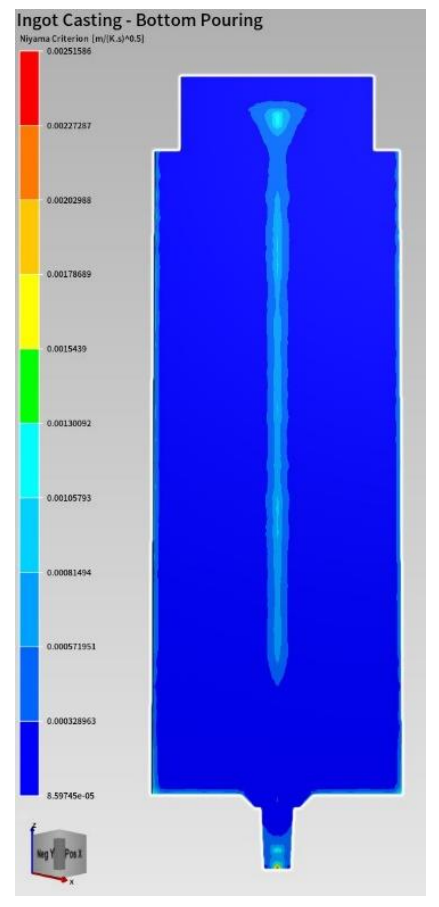

THERCAST

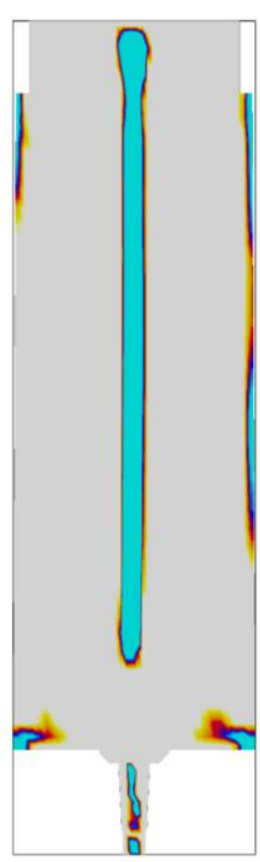

MAGMA

Figure 5 Microporosity formation prediction acc. to the Niyama criterion

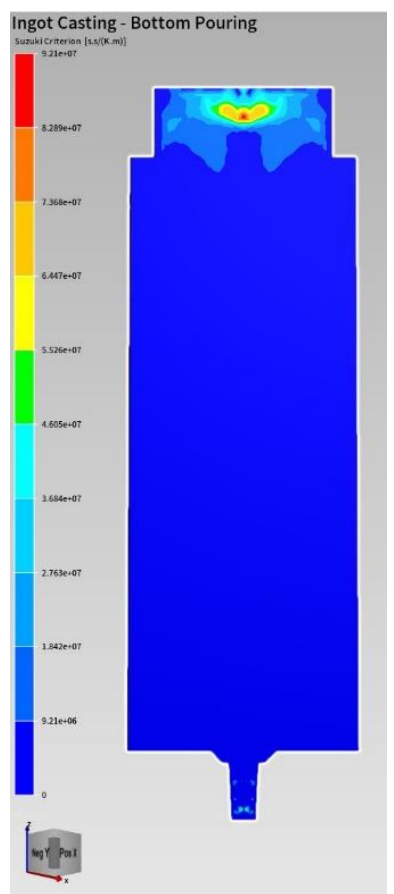

THERCAST

Figure 6 A-segregation formation prediction acc. to the Suzuki criterion

\section{CONCLUSION}

The submitted article deals with the study of casting and solidification of a $12 \mathrm{t}$ ingot made of structural steel 42CrMo4 using numerical simulations. The work was performed using two different software solutions THERCAST and MAGMA. The possibilities of each software tool in filling and solidification of steel were assessed, including the prediction of ingot defects such as porosity, shrinkage cavities or shrinkage porosities; the proportion of liquid phase, segregation of selected elements and solidification time were also monitored.. The achieved pilot results showed that although both compared simulation software tools are based on a certain discretization of the continuum, THERCAST (FEM) in comparison to MAGMA (FDM) showed a more accurate geometric description of the model and representation of boundary conditions with more complex shapes of the integration area. Preliminarily, it appears that THERCAST allows easier use of higher types of approximation of the sought function in order to improve the accuracy of the solution. MAGMA uses cubeshaped elements, which can lead to poorer approximation of the shapes of the simulated body in case the 
body is not rectangular. Finding a suitable cell size was more difficult within the presented simulation task. If a part of the model needs to be defined more precisely, THERCAST software can achieve this with local refinement, while places with simple geometry can be described using a coarser mesh. On the other hand, to improve accuracy with the MAGMA software, it is necessary to increase the number of elements in the entire volume of the model or at least in the section plan, which may result in theoretically higher required computational power with the same accuracy as with THERCAST. Unlike MAGMA in the basic version, THERCAST has the possibility to simulate various behaviours of the material based on one geometric model, including, e.g., strain, stress, density.

\section{ACKNOWLEDGEMENTS}

„This project was created as part of the drawing and use of institutional support for Long-term and conceptual development of a research organization in 2021, provided by the Ministry of Industry and Trade of the Czech Republic".

\section{REFERENCES}

[1] VANNIER, I.; COMBEAU, H.; LESOULT, G. Numerical model for prediction of the final segregation pattern of bearing steel ingots. Materials Science and Engineering: A. 1993, vol. 173, pp. 317-321.

[2] COMBEAU, $\mathrm{H}$. et al. Prediction of macrosegregation in steel ingots: Influence of the motion and the morphology of equiaxed grains. Metallurgical and materials transactions B. 2009, vol 40. pp. 289-304.

[3] NOVÁ, I., KOSEK, P., ŠMRHA, J. Slévárenské simulační programy. MM Průmyslové spectrum. 2005, vol. 11.

[4] MARTÍNEZ, J. et al. Comparative between FEM models for FDM parts and their approach to a real mechanical behaviour. Procedia Engineering. 2013, no. 63, pp. 878-884.

[5] TKADLEČKOVÁ, M. et al. Testing of numerical model settings for simulation of steel ingot casting and solidification. In: Proceedings of the 20th Anniversary International Conference on Metallurgy and Materials METAL. 2011, pp. 61-67.

[6] SI, HO-MUN; CHO, CHONGDU; KWAHK, SI-YOUNG. A hybrid method for casting process simulation by combining FDM and FEM with an efficient data conversion algorithm. Journal of Materials Processing Technology. 2003, vol. 133. pp. 311-321.

[7] TRANSVALOR [Software]. THERCAST ${ }^{\circledR}$, THERCAST reference documentation: Part 1: Ingot Casting, 2020. [2021-03-31]

[8] MAGMASOFT [Software]. MAGMASOFT $®$ MAGMA GmbH, Aachen, Germany. MAGMA reference documentation [2021-03-31]. 